\title{
Perspectivas futuras del marketing. Análisis y posibilidades para el mercado guayaquileño
}

\section{Future prospects of marketing. Analysis and possibilities for the Guayaquil market}

Nicolas Armando Sumba Nacipucha

Universidad Politécnica Salesiana, Ecuador

Jorge Manuel Cueva Estrada

Universidad Politécnica Salesiana, Ecuador

Roberto David López Chila

Universidad Politécnica Salesiana, Ecuador

Autor para correspondencia: nsumba@ups.edu.ec, jcueva@ups.edu.ec, rlopezch@ups.edu.ec

Fecha de recepción: 23 de Enero de 2018 - Fecha de aceptación: 25 de julio de 2018

Resumen: La incursión de nuevas tecnologías en el entorno empresarial presenta a las organizaciones la posibilidad de crear nuevos canales de comunicación que permitan una interacción bidireccional con el cliente. El presente estudio se planteó como objetivo analizar nuevas perspectivas del marketing empleando tecnologías como el internet de las cosas, big data e internet de alta velocidad, para lo cual es necesario determinar si el mercado dispone de los elementos básicos para la implementación de nuevas estrategias de marketing que empleen las tecnologías emergentes. Para ello se empleó el método Delphi y se utilizó herramientas de investigación como la observación, entrevistas a expertos, y encuestas a una muestra de la población guayaquileña para identificar los hábitos de los clientes en lo referente a transacciones de comercio electrónico. El análisis realizado permite manifestar que las perspectivas futuras del marketing habilitarán a las empresas de nuevos canales comunicación que deberá ser empleados con contenido personalizado para cada segmento específico de clientes, y que a su vez permita obtener información de sus clientes en tiempo real para el diseño de productos y servicios personalizados.

Palabras Claves: big data; comercio electrónico; internet de las cosas; personalización de productos

\begin{abstract}
The incursion of new technologies in the business environment presents to the organizations the possibility of creating new channels of communication that allow a bidirectional interaction with the client. The present study aimed to analyze new perspectives of marketing using technologies such as the internet of things, big data and high-speed internet, for which it is necessary to determine if the market has the basic elements for the implementation of new strategies of Marketing that employ emerging technologies. For this purpose, the Delphi method was used and research tools such as observation, interviews with experts, and surveys of a sample of the Guayaquil population were used to identify the habits of the clients in relation to e-commerce transactions. The analysis shows that future marketing prospects will enable companies of new communication channels to be employed with personalized content for each specific segment of customers, and in turn to obtain information from their customers in real time for the design of personalized products and services.
\end{abstract}

Key words: big data; e-commerce; internet of things; product customization 


\section{Introducción}

Prada (2016) concluye que la importancia de las TIC desde un punto de vista estratégico empresarial, reside en identificar las necesidades de los clientes, así como información proveniente de otros actores del entorno empresarial. En este sentido varias investigaciones señalan a las TIC y a sus procesos derivados de innovación e inversión como un nuevo factor de productividad para las organizaciones. (Vilaseca y Torrent, 2006; Jorgenson y Vu, 2007; DíazChao, 2010; González-Gallego, Soto-Acosta, Trigo, Molina-Castillo, Varajão; 2010).

Las organizaciones comerciales desde su génesis deben establecer canales de comunicación con otras empresas, proveedores, conocer de sus competidores, así como con sus clientes, siendo este el punto de inicio para la comunicación comercial. Marín y Lozano (2017) definen que la comunicación empresarial es la que establecen las empresas para relacionarse con sus consumidores y cuyo objetivo radica en lograr el consumo de productos o uso de servicios que oferta la organización al mercado.

La cuestión de esta investigación surge de la relevancia de la comunicación comercial y las TIC en el contexto empresarial actual, la cual instala una interrogante ¿Cómo las organizaciones comerciales pueden adaptar las nuevas tecnologías a sus labores de promoción y marketing?

Gómez Nieto (2017) afirma que la saturación de los medios tradicionales obliga a los especialistas en marketing a buscar soluciones novedosas a problemas de comunicación entre la empresa y el cliente. Es menester de la presente investigación identificar los posibles usos de las nuevas tecnologías en el campo del marketing en las organizaciones comerciales. Para el logro de este objetivo se describe la forma en que los objetos, el internet y las apps interactúan con el cliente y a la vez recolectan datos que serán procesados por técnicas de big data, la misma que se convertirá en una fuente inagotable de información para los líderes empresariales.

La relevancia de la investigación radica en describir el contexto actual en el que las organizaciones comerciales desarrollan sus actividades y como estas deben adaptarse a ese contexto, para ello deberán adoptar las tecnologías emergentes para sus labores de marketing, así como atender a sus clientes tecnológicos que dirigen su atención a una variedad de pantallas digitales.

\section{Fundamentos teóricos}

\section{Tic, marketing y comercio}

El internet y la tecnología móvil han propiciado nuevos canales de comunicación, siendo utilizados por las organizaciones para fortalecer la relación cliente $\leftrightarrow$ empresa. Slusarczyk y Morales (2016) afirman que las tecnologías de la información y la comunicación TIC son una fuente constante de información para la organización, convirtiéndose en un recurso estratégico para las organizaciones actuales que sustenta la toma de decisiones.

El uso constante de equipos digitales por parte de consumidores y organizaciones comerciales, obligó a los expertos en marketing a idear nuevas utilidades para estas tecnologías. 
Arias (2015) indica que las acciones del marketing tradicional deben ser adaptadas a medios digitales alcanzando y mejorando las utilidades de la comercialización tradicional. Mientras Sainz de Vicuña (2015) indica que el marketing digital utiliza de manera correcta y masivamente las tecnologías digitales desarrollando y fortaleciendo la comunicación cliente $\leftrightarrow$ empresa.

Se tiende a confundir que los conceptos de marketing digital y marketing online son iguales, sin embargo, la diferencia radica en que este último añade valor al concepto de marketing digital utilizando el internet como medio de comunicación e interacción con los clientes a través de medios digitales. Olmo y Fondevila (2014) comparten lo expuesto anteriormente indicando que el marketing online se "refiere a la utilización de internet con finalidades de marketing".

Con la combinación del marketing digital y marketing online aparece el comercio electrónico el cual tiene como objetivo concretar la adquisición de productos o servicios ofertados por las organizaciones a través de plataformas digitales de acceso mundial, siendo este proceso una tendencia emergente en la sociedad actual.

La Organización Mundial del Comercio OMC (2016) define al comercio electrónico como producción, publicidad venta y distribución de productos o servicios utilizando telecomunicaciones e internet, adicionalmente indica que el comercio electrónico puede dividirse en ventas y compras electrónicas. A continuación, se presenta un gráfico que resume estos conceptos. La figura 1 muestra un esquema de los conceptos inmerso en el marketing digital.

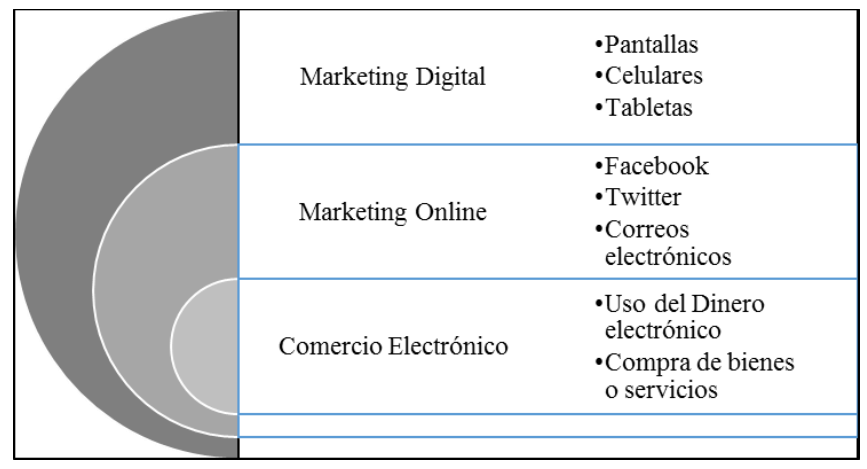

Figura 1 - Conceptos inmersos en el marketing digital Fuente: Elaborado por los autores.

\section{Las empresas y un marketing responsable}

Antes del establecimiento de prácticas que involucran el marketing online y el comercio electrónico, las empresas causaban un serio impacto ambiental a medida que desarrollaban sus diferentes estrategias publicitarias, las cuales incluían el uso en demasía del papel y el plástico para el diseño y creación de los elementos que utilizarían en sus campañas de marketing y publicidad.

Este proceder sumado a la contaminación ambiental despierta la consciencia mundial obligando a las organizaciones a buscar nuevas formas de hacer marketing. Campello, Lorenzo y Plaza (2011) afirman que la aparición de la consciencia ecológica en los consumidores ha causado cambios en la fabricación de los productos, así como en la forma de publicitarlos. Años 
después Martínez Valverde (2015) confirma lo expuesto anteriormente indicando: que el factor ecológico influye en los consumidores quienes ahora buscan productos y servicios de empresas que no perjudiquen el entorno.

En este contexto aparece el concepto de marketing verde, también conocido como marketing ecológico, Martínez Valverde (2015) indica que es una combinación de variables ambientales y variables propias del marketing, precio, calidad, disponibilidad, servicio y diseño para las actividades de venta y desarrollo de productos o servicios. Con el objetivo de atraer el interés de los consumidores preocupados por la preservación del medio.

Entre las empresas se populariza el envío masivo de publicidad vía correo electrónico, uso en redes sociales como medio publicitario y la creación de páginas web institucionales. El uso de estas herramientas tecnológicas crea nuevos canales de comunicación entre la empresa $\leftrightarrow$ el cliente, además de minimizar el uso de papel y plástico, elementos contaminantes del entorno.

En este contexto aparecen herramientas tecnológicas como los códigos QR permitiendo a través de un escaneo rápido con un dispositivo digital el acceso a información multimedia. Esta utilidad permitió a las organizaciones orientar sus usos al marketing. En este sentido Cueva y Sumba (2016) indican que los códigos QR permiten "establecer vínculos directos de interrelación con el cliente y a su vez amigables con el entorno", esta idea es complementada por Silva (2016) quien identifica a los códigos QR como una herramienta aplicable a cualquier empresa en el ámbito del marketing y la publicidad.

\section{Nuevas tecnologías aplicables al marketing}

La nanotecnología en la informática y la electrónica se aplica según Fidalgo, Fernández Pérez y Fernández Fernández (2016) en ordenadores muy pequeños, pero con alta capacidad de almacenamiento y velocidad de procesamiento, características aplicables a dispositivos móviles. Estas características combinadas con el internet de alta velocidad han permitido vincular equipos electrónicos a objetos de uso diario, añadiéndoles valor, esta fusión dio paso al denominado internet de las cosas.

Bessis y Dobre (2014) describen que el internet de las cosas "integra, recolecta información y ofrece servicios a un muy diverso espectro de cosas físicas usadas en diferentes dominios" mientras que la Organización para la Cooperación y el Desarrollo Económico (2016) señala que el internet de las cosas "engloba todos los dispositivos y objetos cuyo estado puede consultarse o modificarse a través de internet, con o sin participación activa de personas".

La información se ha convertido en un recurso estratégico para las sociedades comerciales actuales, Aportela (2015) hace una reflexión acerca de la importancia de la comunicación en las organizaciones "Las empresas que mejor asimilen las informaciones disponibles, tanto en su ambiente interno como externo, tendrán mayores oportunidades de supervivencia y niveles de competitividad más elevados.", para ello deberán utilizar sistemas de información que les permita gestionarla y analizarla. 
Desde el punto de vista del marketing, Kotler y Armstrong (2012) manifiestan que la importancia de la información obtenida a través de la investigación de mercados radica en la forma en que será utilizada, siendo la satisfacción y la relación con el cliente a largo plazo, sus objetivos finales. Este es el punto de inicio que sustenta el marketing de relaciones, que tiene como enfoque brindar soluciones al cliente por encima de la venta de productos. La figura 2 muestra un esquema de transformación de los datos en conocimiento empresarial

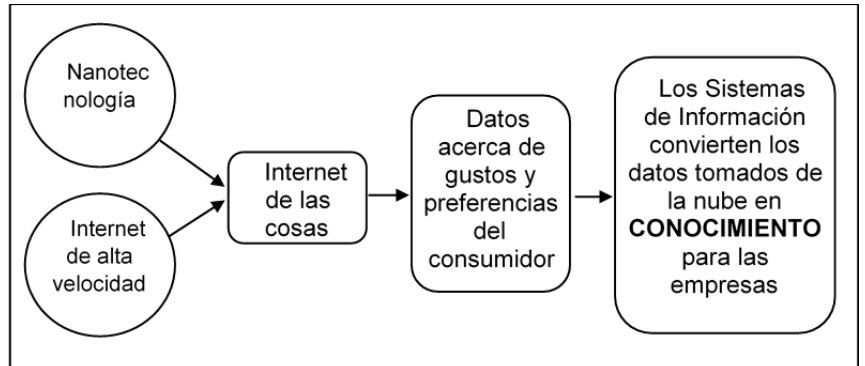

Figura 2 - Transformación de datos provenientes del cliente en conocimiento empresarial.

Las aplicaciones para dispositivos digitales apps se han convertido en una fuente de información del entorno empresarial gracias a su ubicuidad, estando presentes en diferentes aspectos del entorno. Vizuete (2012) explica que los usuarios interactúan con una gran variedad y tipos de aplicaciones móviles, tanto en el ámbito personal como laboral. La facilidad de interacción con los usuarios y la ubicuidad dieron paso a la creación de nuevas formas de uso de las apps por parte de las organizaciones comerciales. En este sentido Palos, Martín y Folgado (2016) señalan que las apps se han convertido en uno de los medios publicitarios con mayor previsión de crecimiento en los próximos años.

Las diferentes aplicaciones instaladas en dispositivos móviles generan enormes conjuntos de datos para las empresas, lo cual da paso a la aplicación de técnicas de big data, encargadas de almacenar, procesar y extraer información oculta en grandes volúmenes de datos y ponerla a servicio de los usuarios, pudiendo ser utilizada en diferentes ámbitos: comercio, salud, educación, ciencia y gobierno. (Dhar, 2014; Mohanty, 2015, Bughin, 2016).

La inteligencia artificial se aprovecha de la gran cantidad de conocimiento que se puede obtener a través de la big data, en este sentido se observan aplicaciones de la inteligencia artificial en diferentes campos (Santana, Costaguta, Missio, 2014; Flores, Quelopana, 2016; Núñez, Rey y Caridad, 2016). En el campo del marketing, los algoritmos de inteligencia artificial son utilizados para razonar, crear y pensar en el desarrollo de nuevos productos y líneas de venta, así como para optimizar y mejorar los servicios de atención al cliente, todo esto sustentado en tendencias del mercado, gustos y preferencias del consumidor, estas aplicaciones son soportadas por lo expuesto por Benítez, Escudero, Kanaan y Masip (2013) quien indica que la inteligencia artificial emula algunas de las facultades intelectuales humanas en sistemas artificiales.

Martin (2015) observa la omnipresencia de los dispositivos con pantallas digitales en el diario vivir, las comunicaciones, el trabajo e incluso la diversión de las personas que integran la sociedad. Esto ha provocado que los actores del entorno empresarial (clientes, competidores, proveedores y empleados) desarrollen sus actividades diarias interactuando con diferentes pantallas. 
Mendieta (2013) indica que el efecto multipantalla implica el uso de la computadora, el televisor y dispositivos móviles, como tabletas y teléfonos inteligentes, para acceder a una variedad de contenidos por parte de los consumidores. Esta tendencia es afianzada en la investigación de Guallar y Orduña (2014) quienes indican que los consumidores inician la búsqueda de información de algún producto en un tipo de dispositivo para luego continuar en otro, esto gracias al efecto multipantalla, este proceso continúa hasta finalizar con la compra, que en muchas ocasiones no se realiza de manera electrónica.

Lo expuesto anteriormente indica que el efecto multipantalla aplicado al marketing no necesariamente concreta el objetivo de venta a través de un dispositivo electrónico, sin embargo, es clave que la información de los productos debe tener omnipresencia en las pantallas digitales. Este comportamiento debe ser aprovechado por las organizaciones comerciales tratando de estar presente en las diferentes pantallas mostrando contenidos interactivos y de interés para el consumidor.

Las organizaciones comerciales a nivel mundial se encuentran en constante desafío: el reto de dominar tecnologías emergentes que gracias a la informática y la telemática se encuentran en continuo desarrollo. Con la aparición de la globalización, las organizaciones comerciales están obligadas a poder adaptarse a la tendencia tecnológica emergente mundial para ser competitivas en mercados globalizados.

Con lo expuesto anteriormente y siendo el marketing una de las funciones primarias en las organizaciones comerciales los autores plantean las siguientes interrogantes: ¿Existe en el mercado guayaquileño las condiciones necesarias para el marketing del futuro? ¿Qué opinan los expertos acerca de la aplicabilidad de nuevas tecnologías en el marketing?

\section{Metodología}

Para alcanzar el objetivo que persigue la investigación, se aplicó la observación como herramienta de investigación para describir nuevas características y usos de tecnologías que podrían ser aplicables al marketing.

También se utilizó el método Delphi como procedimiento para el análisis de la investigación cualitativa, para lo cual en primera instancia se entrevistó a tres expertos en marketing y tecnologías digitales (Grupo A) para considerar su opinión acerca de los temas a tratar en el cuestionario de preguntas dirigidas a un grupo más amplio y diverso de expertos (Grupo B), quienes se desenvuelven en diferentes áreas: publicidad, marketing digital, investigación de mercados, telefonía móvil y tecnologías de la información y de la comunicación. El cuestionario aplicado al Grupo B contenía ocho preguntas abiertas y este es aplicado vía correo electrónico considerando las diferentes ocupaciones de los especialistas. La intención de este cuestionario es invitar a los expertos a extenderse en los temas tratados, considerando que sus opiniones permiten abordar el tema de investigación desde diferentes puntos de vistas, lo cual sustenta los resultados del análisis final.

Posteriormente se extraen las opiniones más recurrentes de los expertos del Grupo B y se procede a elaborar un segundo cuestionario con preguntas politómicas de única respuesta, el cual 
se aplica a los mismos especialistas del Grupo B para de esta forma, contar con ideas estructuradas de los expertos sobre la aplicación de las nuevas tecnologías en el marketing.

Para complementar los resultados obtenidos con el método Delphi se realizó un muestreo probabilístico aleatorio, el cual fue desarrollado en diferentes ubicaciones geográficas de la ciudad de Guayaquil. La población de estudio es 1'154.229 guayaquileños, las cuales, según el Instituto Nacional de Estadística y Censo (INEC) en el año 2017 cuentan con condiciones adecuadas de empleo. Para un $95 \%$ de confianza el tamaño de la muestra es de 384, sin embargo, el cuestionario fue aplicado a 395 personas. La aplicación de este cuestionario permitirá describir las prácticas de comercio electrónico del mercado guayaquileño.

\section{Resultados}

Como derivación principal del proceso de observación realizado en la Feria Mundial de Tecnología de Consumo CES desarrollado desde el 5 al 8 de enero de 2017 en las Vegas, se puede afirmar que en los electrodomésticos de línea blanca existe una creciente tendencia para amalgamar conceptos mencionados en el marco teórico: la nanotecnología, pantallas digitales e internet de alta velocidad. A continuación, se resume y describe nuevas características encontradas en productos electrónicos durante el proceso de observación.

Refrigeradoras: tiene como característica básica crear un ambiente frio en su interior para conservación y preservación de alimentos. Mientras que actualmente a las funciones básicas se le agrega una pantalla digital con conexión a internet la cual permite el acceso a contenido multimedia en línea, permite crear listas de compras. Incluye sensores para detectar alimentos calientes y automáticamente bajar la temperatura del interior. Una cámara digital que toma fotografías del interior del refrigerador, esto como ayuda al momento de realizar las compras.

Lavadoras de ropa: sus procesos básicos son el lavado y secado de ropa. pero actualmente se añade la inclusión de una pantalla digital que permite interactuar al usuario con los diferentes programas especializados de lavado pudiendo ser descargados desde internet.

Cocinas: generar calor para la cocción de los alimentos ha sido desde siempre la función básica de este electrodoméstico a esta utilidad se le añade la inclusión de sensores que detectan el peso del alimento y en función de eso establecer el tiempo y la temperatura adecuada para su cocción. Pantalla digital con acceso ha contenido multimedia y recetarios además de la opción de descargar diferentes programas de cocción de alimentos. Alertas enviadas a celulares indicados el fin del ciclo de cocción.

Celulares o tablets: equipo de comunicación. Que ahora incluye alta calidad de resolución en sus cámaras fotográficas. Mayor velocidad y facilidad de navegación por internet. Altas capacidades de almacenamiento y procesamiento de datos. Sensores y comunicación con electrodomésticos.

Ropa o Calzado: vestir el cuerpo humano ha sido su función básica mientras que en la actualidad las prendas de vestir incluyen sensores que capturan información de deportistas como cantidad de kilómetros recorridos, peso corporal, o niveles de agotamiento físico. 
Como puede notarse según lo expuesto es de uso común en estas nuevas tecnologías la conexión de los objetos al internet, también la incorporación de pantallas digitales las cuales incrementan el efecto multipantalla y por último la creación de aplicaciones, descargadas de manera gratuita a celulares o tablets, esta aplicación permite al usuario interactuar con la marca y a esta última recibir información pertinente del cliente transformado luego en conocimiento. La figura 3 muestra un esquema que resume el desarrollo de los nuevos electrodomésticos.

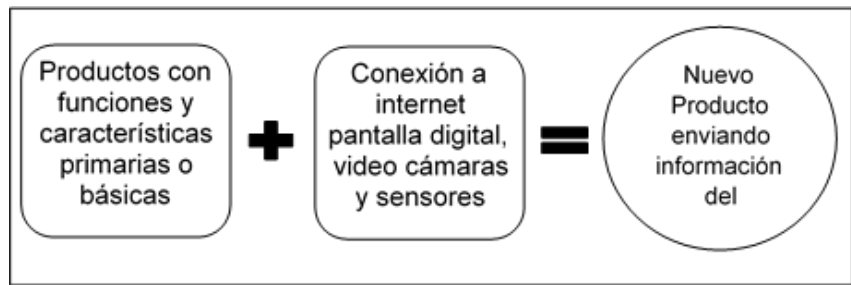

Figura 3 - Transformación de los productos básicos a productos conectados a internet. Fuente: Elaborado por los autores.

Las posturas de las entrevistas a expertos se resumen en las siguientes ideas:

En lo que respecta al internet de las cosas, los objetos se convertirán en nuevas pantallas digitales, permitiendo al marketing utilizar dichos objetos como medios de comunicación e interacción con el cliente. En este aspecto, los expertos recomiendan que las nuevas pantallas se centren en presentar información de interés del consumidor, para evitar el hostigamiento de los clientes, como el generado por el envío de correos masivos que no eran bien recibidos por los consumidores. Otros aportes manifiestan que la incorporación de pantallas en la diversidad de objetos consolidará el denominado efecto multipantalla en la sociedad.

Otro consenso generado por la opinión de los expertos es la utilidad que las empresas podrán darle a la gran cantidad de información generada por el internet de las cosas y procesada mediante técnicas de big data. De esta forma las empresas contarán con conocimiento específico de sus clientes para la elaboración de nuevos productos o servicios cada vez más personalizados. Los expertos concluyen que el futuro de la producción de las empresas no es la fabricación de productos en serie sino la fabricación de productos adaptados a diferentes nichos y mercados globalizados.

Para los expertos la ubicuidad del marketing, se manifestará al desarrollarse en tiempo real, ofreciendo productos o servicios en el momento y lugar en el que el cliente los necesite, debido a la disponibilidad de internet de alta velocidad y que debe ser complementado por la apuesta de las empresas en el ámbito de la Investigación, Desarrollo e Innovación I+D+I, lo cual permitirá entre otras cuestiones, la creación de novedosas aplicaciones app para dispositivos digitales destinadas a satisfacer las necesidades concretas del consumidor.

Los expertos también expresaron concordancia de opiniones al manifestar que las etiquetas de los productos deberán convertirse en un canal de acceso a información de significancia para el cliente, lo cual será logrado mediante la combinación de tecnologías como el código QR, chips NFC, realidad aumentada y apps móviles. Según los expertos, esta combinación permitirá brindar a los clientes una diversidad de utilidades tales como conocer los 
lugares más cercanos de compra del producto, recetarios, guías de instalación, procesos de fabricación y diferentes usos y recomendaciones acerca del producto.

\section{Resultados de las encuestas}

Con participación similar de hombres y mujeres se aplica la encuesta a 395 habitantes de la ciudad de Guayaquil, con edades comprendidas de 18 a 64 años, de estos la mayor cantidad de encuestados se sitúa en edades que oscilan de 25 a 32 años, mientras que su nivel predominante de educación es la universitaria. Con estas características los resultados de la encuesta son los siguientes:

El 66.6\% de los encuestados manifestaron incomodidad por recibir publicidad en medios digitales que no es de su interés particular. Por otra parte, el $48.6 \%$ de los encuestados indicaron estar interesados en recibir promociones de productos o servicios en dispositivos electrónicos de manera personalizada. En la Gráfica 1 se muestran las cantidades y porcentajes de encuestados que afirman haber realizado transacciones comerciales por internet."

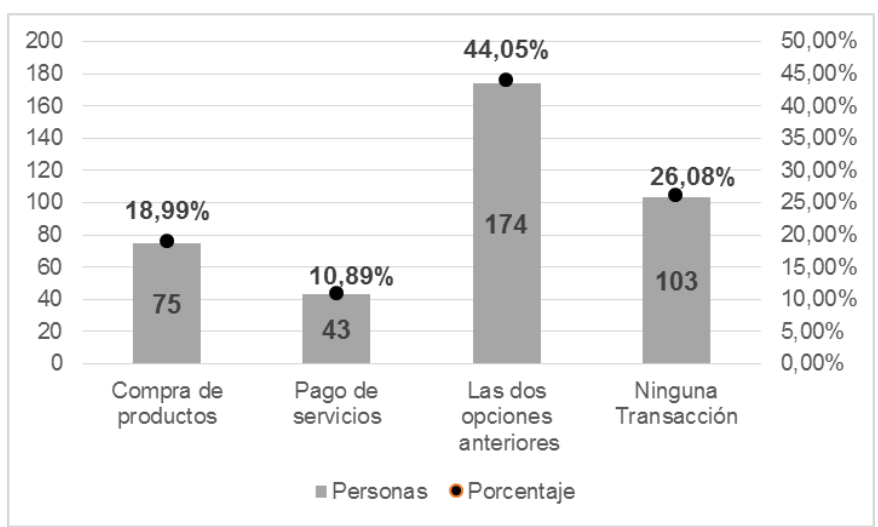

Gráfica 1 - Porcentaje de personas que han realizado transacciones por internet.

Fuente: Elaborado por los autores.

Un dato relevante con respecto a la publicidad y la promoción en medios digitales es que el 71,2\% de los encuestados que han realizado transacciones comerciales por internet, afirmaron haber adquirido algún producto o servicio, influenciados por un anuncio publicitario observado en un dispositivo digital.

Para los encuestados, el orden de importancia de los motivos para realizar transacciones comerciales por internet es la siguiente: en primer lugar, el ahorro de tiempo y esfuerzo, seguido de ofertas y precios especiales continuando con beneficios y acumulación de puntos para futuras compras y en último lugar, preferencia del internet como canal de comunicación.

En lo que respecta a la forma de iniciar la recopilación de información para la adquisición de un nuevo producto o servicio, el 44,52\% de los encuestados afirmaron que su búsqueda inicia en internet en detrimento de la visita a tiendas físicas, llamadas telefónicas y consultas a amigos o conocidos. En contraparte, un hallazgo importante es que el 54,79\% de los encuestados terminan su proceso de compra en tiendas físicas. 
Por otra parte, de las personas encuestadas que no han realizado transacciones comerciales por internet, el 57,3\% manifiesta sentir inseguridad en la forma de pago, el 39,8\% no dispone de tarjeta de crédito u otras formas de pago, mientras que otros motivos en porcentajes más bajos fueron: desconocimiento para realizar la transacción, desconfianza sobre la calidad del producto y ausencia de opciones de comercio electrónico en páginas web empresariales.

Un resultado a considerar es: el 93.9\% de los encuestados manifestaron su completa satisfacción al encontrar productos y servicios desarrollados o diseñados considerando sus opiniones, gustos y preferencias.

\section{Conclusiones}

La sociedad digital emergente que se ha instaurado desde hace varios años atrás presenta continuos avances tecnológicos que marcan el camino que recorren las personas y las sociedades comerciales en su cotidianidad. En este sentido las empresas afrontan el desafío de emplear las nuevas tecnologías a fin de brindar nuevos servicios y mejorados productos de calidad a sus clientes, adaptándose a un mundo globalizado y cambiante continuamente.

La integración de nuevas tecnologías que insertarán pantallas digitales en los objetos de uso cotidiano del cliente y que estarán disponibles para el entorno empresarial, sumado al internet de alta velocidad, dotarán a las empresas de nuevos canales de comunicación, los cuales deberán ser utilizados de manera óptima con contenido personalizado y de calidad para cada uno de los clientes en los micro segmentos de mercado. La intención de esta personalización de contenidos es evitar el hostigamiento del cliente por recibir publicidad generalizada y más aun teniendo en cuenta que la tecnología permitirá a los consumidores evadir publicidades que no sean de su interés.

El internet de las cosas, el big data y el acceso a internet de alta velocidad pondrá a disposición del marketing información en tiempo real del cliente y de su entorno para el diseño y elaboración de nuevos productos o servicios. Estas tecnologías a la vez abren una nueva posibilidad para las organizaciones, la atención al cliente en tiempo real. Lo expuesta anteriormente obliga a pensar en la posible omnipresencia de las organizaciones comerciales, lo cual deja la puerta abierta a futuras investigaciones referentes a este tema.

Si bien es cierto el marketing empresarial dispondrá de conocimiento en tiempo real sobre los clientes y que en consecuencia buscará satisfacer a cada uno de ellos mediante productos y servicios personalizados, se hacen necesario revisar si las empresas podrán llevar a cabo este cometido considerando que tradicionalmente las organizaciones han realizado su producción en serie para optimizar costos y materia prima. Esta reflexión deja la puerta abierta a nuevas investigaciones que ahonden en la capacidad y posibilidad de incluir nuevas tecnologías que permitan a las empresas alcanzar altos niveles de personalización tanto en productos como en servicios. 


\section{Bibliografía}

Aportela Rodríguez, I. M. (2015). La información como recurso estratégico en las empresas de base tecnológica. Revista General de Información y Documentación, 25(2). doi:10.5209/rev_rgid.2015.v25.n2.51238

Arias, A. (2015). Las nuevas tecnologías y el marketing digital. 2da Edición. Recuperado de http://goo.gl/Nt8SDI

Benítez, R., Escudero, G., Kanaan, S. y Masip, D. (2013). Inteligencia Artificial Avanzada. Barcelona, España: Editorial UOC

Bessis, N., y Dobre, C. (Eds.). (2014). Big Data and Internet of Things: A Roadmap for Smart Enviroments. Suiza: Springer International Publishing.

Bughin, J. (2016). Big data, Big bang? Journal of Big Data, 3(1). doi:10.1186/s40537-015-00143

Campello, M., Lorenzo, F. y Plaza, A. (2011) Análisis de la evolución de la publicidad ecológica en prensa (2005-2007). Miguel Hernández Communication Journal,1 (2), 13-39.

Cueva, J. y Sumba, N. (2016). Marketing con códigos QR en las sociedades comerciales: entre la expectativa y la realidad, propuesta para una adecuada implementación. En R. Ayala, J. Llerena y P. Parra (Coordinadores) (2016). Segundo Congreso Salesiano de Ciencia, Tecnología e Innovación para la Sociedad. Memoria Académica (pp. 103-116).Quito, Ecuador: Editorial Universitaria Abya-Yala.

Del Olmo, J. y Fondevila, J. (2014). Marketing digital en la moda. Madrid, España: Ediciones internacionales universitarias, S.A.

Dhar, V. (2014). Why Big Data = Big Deal. Big Data, 2(2), 55-56. doi:10.1089/big.2014.1522

Díaz-Chao, A. (2010). ¿Pueden el uso de las TIC y los activos intangibles mejorar la competitividad? Un análisis empírico para la empresa catalana. Estudios de economía aplicada, 28(2), 477.

Fidalgo, J., Fernández Pérez, M. y Fernández Fernández, N. (2016). Tecnología Industrial II. Madrid, España: Ediciones Paraninfo.

Flores, V. y Quelopana, A. (2016). An Intelligent System Prototype to support and sharing diagnoses of maligned tumors, based on personalized medicine philosophy. Revista Iberoamericana de Inteligencia Artificial 19(58), 17-22. doi: 10.4114/ia.v19i58.1155 
Gonzálvez-Gallego, N., Soto-Acosta, P., Trigo, A., Molina-Castillo, F. J., \& Varajão, J. (2010). El papel de las TIC en el rendimiento de las cadenas de suministro: el caso de las grandes empresas de España y Portugal. Universia Business Review, 4(28).

Gómez Nieto, B. (2017). Fundamentos de la publicidad. Madrid, España: Esic Editorial.

González Fernández-Villavicencio N. (2014). El efecto ROPO y las bibliotecas. En. J. Guallar (Director) y E. Orduña (Cood). Análisis de tendencias en información y documentación 2014. (pp. 334-341). Barcelona, España: Editorial UOC.

Gómez, B. (2016). Mass customization Las claves de la personalización masiva. 3era Edición. Barcelona, España: Gestión Editorial.

Instituto Nacional de Estadísticas y Censos. INEC. (2015). Principales indicadores de actividades de ciencia, tecnología e innovación. Recuperado de goo.gl/gvmj4n

Instituto Nacional de Estadísticas y Censos. INEC. (2017). Tecnologías de la información y comunicaciones (TIC'S) 2016. Recuperado de goo.gl/oVNqVz

Instituto Nacional de Estadísticas y Censos. INEC. (2017). Encuesta nacional de empleo, desempleo y subempleo. Recuperado de goo.gl/g1aQch

Jorgenson, D.W.; VU, K. (2007): "Information technology and the World growth resurgence", German Economic Review, Vol. 8, Núm. 2, pp.122-145.

Kotler, P y Armstrong, G. (2012). Marketing Decimocuarta edición. México D.F., México: Pearson Educación.

Marín, P. y Lozano, J. (2017). La comunicación de marketing en la empresa de distribución española Mercadona. Retos, $n^{\circ}$ 13, vol. VII, 9-26. doi: http://dx.doi.org/10.17163/ret.n13

Martínez Valverde, J. (2015). Marketing en la actividad comercial. Madrid, España: Ediciones Paraninfo.

Mendieta, A. (2013). Tendencias de vanguardia en la comunicación. Madrid, España: Visión Libros.

MMA (2010): “3er Estudio de Inversión en Marketing y Publicidad Móvil en España 2010”. Recuperado de http://goo.gl/3Ozcda

Mohanty, H. (2015). Big Data: An Introduction. Big Data, 1-28. doi:10.1007/978-81-322-2494$5 \_1$

Núñez, J., Rey, F. y Caridad, J. (2016). Artificial Intelligence (AI) techniques to analyze the determinants attributes in housing prices. Revista Iberoamericana de Inteligencia Artificial 19(58), 23-38. doi: 10.4114/ia.v19i58.1156 
Organización para la Cooperación y el Desarrollo Económicos. (2016). Perspectivas de la OCDE sobre la economía digital 2015. Ciudad de México, México: Organización para la Cooperación y el Desarrollo Económicos

Organización Mundial de Comercio OMC (2016). Las pymes en el comercio internacional: hechos estilizados. Recuperado de http://goo.gl/3U3cmb

Palos Sánchez, P. R., Martín Cumbreño, E., \& Folgado Fernández, J. A. (2016). Factores condicionantes del marketing móvil: estudio empírico de la expansión de las apps. El caso de la ciudad de Cáceres. Revista de Estudios Económicos y Empresariales (28), pp. 37-72.

Prada Ospina, R. (2016). Las TIC, factor de efectividad en la aplicación del mercadeo digital en negocios de retail. Revista EAN, (80), 105. doi:10.21158/01208160.n80.2016.1460

Rodríguez-Carmona, L. M. (2012). Claves para valorar el marketing móvil. Vivat academia, (117E), 51-62.

Sainz de Vicuña, J. (2015). El plan de marketing digital en la práctica. Madrid, España: Esic Editorial. Santa Fé, Argentina: Centro de Publicaciones, Secretaria de Extensión Universidad Nacional del Litoria.

Santana, P., Costaguta, R. y Missio, D. (2014). Aplicación de Algoritmos de Clasificación de Minería de Textos para el Reconocimiento de Habilidades de E-tutores Colaborativos. Revista Iberoamericana de Inteligencia Artificial 17(53), 57-67

Silva Ordoñez, F., Medina Chicaiza, P., \& Sarzosa Rivera, S. (2016). Estudio De Factibilidad Para Publicitar Productos Empresariales Con Códigos QR. 3C Tecnología_Glosas de Innovación Aplicadas a La Pyme, 5(3), 1-19. doi:10.17993/3ctecno.2016.v5n3e19.1-19

Slusarczyk Antosz, M., \& Morales Merchán, N. H. (2016). Análisis de las estrategias empresariales y de las TIC. 3C Empresa Investigación y Pensamiento Crítico, 05(01), 2946. doi:10.17993/3cemp.2016.050125.29-46

Vilaseca, J.; Torrent, J. (a2006): "TIC, conocimiento y crecimiento económico. Un análisis empírico, agregado e internacional sobre las fuentes de la productividad", Economía Industrial, Núm. 360, pp. 41-60

Vizuete, M. (2012). Analizar el aporte de las soluciones de aplicaciones móviles para el comercio electrónico en la ciudad de Quito. (Tesis de maestría). Recuperado de:

http://dspace.ups.edu.ec/bitstream/123456789/4901/1/UPS-QT03467.pdf 